\title{
On the security of some password-based key agreement schemes
}

\author{
Qiang Tang and Chris J. Mitchell \\ Information Security Group \\ Royal Holloway, University of London \\ Egham, Surrey TW20 0EX, UK \\ \{qiang.tang,c.mitchell\}@rhul.ac.uk
}

23rd May 2005

\begin{abstract}
In this paper we show that two potential security vulnerabilities exist in the strong password-only authenticated key exchange scheme due to Jablon. Two standardised schemes based on Jablon's scheme, namely the first passwordbased key agreement mechanism in ISO/IEC FCD 11770-4 and the scheme BPKAS-SPEKE in IEEE P1363.2 also suffer from one or both of these security vulnerabilities. We further show that other password-based key agreement mechanisms, including those in ISO/IEC FCD 11770-4 and IEEE P1363.2, also suffer from these two security vulnerabilities. Finally, we propose means to remove these security vulnerabilities.
\end{abstract}

\section{Introduction}

Password-based authenticated key agreement has recently received growing attention. In general, such schemes only require that a human memorable secret password is shared between the participants. In practice, passwordbased schemes are suitable for implementation in a wide range of environments, especially those where no device is capable of securely storing high-entropy long-term secret keys. Password-based key agreement schemes originate from the pioneering work of Lomas et al. [1]. Subsequently many password-based key establishment schemes have been proposed, including by Bellovin and Merritt [2, 3], Steiner et al. [4], Jablon [5, 6], Lucks [7], $\mathrm{Wu}$ [8], Goldreich and Lindell [9], Bellare et al. [10, 11], Bresson et al. [12], Kwon [13], Nguyen and Vadhan [14], Abdalla et al. [15, 16, 17], and Laih et 
al. [18].

However, despite their implementation convenience, password-based key agreement schemes are prone to password guessing attacks, because the users often select their passwords so that they can be easily memorised, which means that they are likely to be much easier to guess than randomly selected passwords. Moreover, for convenience, many users select the same passwords with different partners. For example, in a client-server setting, the client might choose to use the same password with several different servers.

In this paper, we first show that two potential security vulnerabilities exist in Jablon's strong password-only authenticated key agreement scheme [5]. The first password-based key agreement mechanism in [19] and the scheme BPKAS-SPEKE in [20], which are both based on Jablon's scheme, also suffer from one or both of these security vulnerabilities. In fact, other passwordbased key agreement schemes also suffer from these vulnerabilities. Finally, we show how to remove these vulnerabilities.

\section{Description of Jablon's scheme}

In this section, we describe the Jablon scheme. At relevant points we also point out the differences between the Jablon scheme and the first passwordbased key agreement mechanism (in the discrete logarithm setting) in [19], and the scheme BPKAS-SPEKE (in the discrete logarithm setting) in [20].

In the Jablon protocol, the following parameters are made public. $p$ and $q$ are two large prime numbers, where $p=2 q+1$. $h$ is a strong one-way hash function. Suppose a user $(U)$ with identity $I D_{U}$ and a server $(S)$ with identity $I D_{S}$ share a secret password $p w$. When $U$ and $S$ want to negotiate a session key, they first compute $g=p w^{2} \bmod p$.

Note that in the first mechanism of ISO/IEC FCD 11770-4 [19] $g$ is instead computed as $h(p w \| s t r)^{2}$, where str is an optional string. Also, in BPKAS-SPEKE in draft D20 of P1363.2 [20], $g$ is instead computed as $h(\text { salt }\|p w\| s t r)^{2}$, where salt is is a general term for data that supplements a password when input to a one-way function that generates password verification data. The purpose of the salt is to make different instances of the function applied to the same input password produce different outputs. Finally, str is an optional string which it is recommended should include $I D_{S}$.

$U$ and $S$ perform the following steps.

1. $U$ generates a random number $t_{1} \in Z_{q}^{*}$, and sends $m_{1}=g^{t_{1}} \bmod p$ to $S$. 
2. After receiving $m_{1}, S$ generates a random number $t_{2} \in Z_{q}^{*}$, and sends $m_{2}=g^{t_{2}} \bmod p$ to $U$. $S$ computes $z=g^{t_{2} t_{1}} \bmod p$, and checks whether $z \geq 2$. If the check succeeds, $S$ uses $z$ as the shared key material, and computes $K=h(z)$ as the shared key.

3. After receiving $m_{2}, U$ computes $z=g^{t_{2} t_{1}} \bmod p$, and checks $z \geq$ 2. If the check succeeds, $U$ uses $z$ as the shared key material, and computes $K=h(z)$ as the shared key. Then $U$ constructs and sends the confirmation message $C_{1}=h(h(h(z)))$ to $S$.

Note that in both the ISO/IEC FCD 11770-4 and IEEE P1363.2 versions of the mechanism, $C_{1}$ is instead computed as:

$$
C_{1}=h\left(3\left\|m_{1}\right\| m_{2}\left\|g^{t_{1} t_{2}}\right\| g\right),
$$

4. After receiving $C_{1}, S$ checks that the received message equals $h(h(h(z)))$. If the check fails, $S$ terminates the protocol execution. Otherwise, $S$ computes and sends the confirmation message $C_{2}=h(h(z))$ to $U$.

Note that in both the ISO/IEC FCD 11770-4 and IEEE P1363.2 versions of the mechanism, $C_{2}$ is instead computed as:

$$
C_{2}=h\left(4\left\|m_{1}\right\| m_{2}\left\|g^{t_{1} t_{2}}\right\| g\right),
$$

5. After receiving $C_{2}, U$ checks that it equals $h(h(z))$. If the check fails, $U$ terminates the protocol execution. Otherwise, $U$ confirms that the protocol execution has successfully ended.

Finally, note that in the elliptic curve setting the first password-based key agreement mechanism in [19] and the scheme BPKAS-SPEKE in [20] are essentially the same as above, except that $g$ is a generator of the group of points on an elliptic curve.

\section{Security vulnerabilities}

In this section we describe two security vulnerabilities in the Jablon protocol. In addition, we show that the standardised password-based key agreement mechanisms in $[19,20]$ also suffer from one or both of these vulnerabilities.

\subsection{The first security vulnerability}

This security vulnerability exists when one entity shares the same password with at least two other entities. This is likely to occur when a human user chooses the passwords it shares with a multiplicity of servers. Specifically 
we suppose that a client, say $U$ with identity $I D_{U}$, shares a password $p w$ with two different servers, say $S_{1}$ with identity $I D_{S_{1}}$ and $S_{2}$ with identity $I D_{S_{2}}$. A malicious third party can mount the attack as follows.

Suppose $U$ initiates the protocol with an attacker which is impersonating server $S_{1}$. Meanwhile the attacker also initiates the protocol with server $S_{2}$, impersonating $U$. The attacker now forwards all messages sent by $U$ (intended for $S_{1}$ ) to $S_{2}$. Also, all messages sent from $S_{2}$ to $U$ are forwarded to $U$ as if they come from $S_{1}$. At the end of the protocol, $U$ will believe that he/she has authenticated $S_{1}$ and has established a secret key with $S_{1}$. However $S_{1}$ will not have exchanged any messages with $U$. In fact, the secret key will have been established with $S_{2}$.

The above attack demonstrates that, even if the server $\left(S_{1}\right)$ is absent, the attacker can make the client believe that the server is present and that they have computed the same session key as each other. Of course, if $U$ shares the same password with servers $S_{1}$ and $S_{2}$, then $S_{1}$ can always impersonate $U$ to $S_{2}$ and also $S_{2}$ to $U$, regardless of the protocol design. However, the problem we have described in the Jablon scheme applies even when $U, S_{1}$ and $S_{2}$ all behave honestly, and this is not a property that is inevitable (we show below possible ways in which the problem might be avoided).

Based on the descriptions in Section 2, it is straightforward to mount this attack on the first password-based key agreement mechanism in [19]. In fact, this attack also applies to the other key agreement mechanisms in [19]. However, if the identifier of the server is used in computing $g$, e.g. if it is included in the string str, then this attack will fail. The scheme BPKASSPEKE in [20] is thus immune to this attack as long as the recommendation given in [20] to include this identifier in $s t r$ is followed.

\subsection{The second security vulnerability}

This security vulnerability, which applies to a variety of password-based schemes, exists when two instances of the protocol are concurrently executed. One example of circumstances in which such concurrent execution may arise is when the same key agreement protocol and secret key are used by multiple applications. Without loss of generality, we show how the attack works on the Jablon scheme.

Suppose a client, say $U$ with identity $I D_{U}$, shares a password $p w$ with a server, say $S$ with identity $I D_{S}$. If the client simultaneously initiates two instances of the protocol with $S$, then the attacker can mount the attack as follows:

1. In the first protocol instance, suppose that $U$ generates random number $t_{1} \in Z_{q}^{*}$, and sends $m_{1}=g^{t_{1}} \bmod p$ to $S$. In the second protocol 
instance, suppose that $U$ generates random number $t_{1}^{\prime} \in Z_{q}^{*}$, and sends $m_{1}^{\prime}=g^{t_{1}^{\prime}} \bmod p$ to $S$.

The attacker then intercepts and swaps these two messages, i.e., it impersonates $U$ to send $m_{1}$ and $m_{1}^{\prime}$ to $S$ in the second and first protocol instances respectively.

2. In the first protocol instance, after receiving $m_{1}^{\prime}, S$ generates random number $t_{2} \in Z_{q}^{*}$, and sends $m_{2}=g^{t_{2}} \bmod p$ to $U$. $S$ also computes $z^{\prime}=g^{t_{2} t_{1}^{\prime}} \bmod p$ as the shared key material with $U$, and computes $K^{\prime}=h\left(z^{\prime}\right)$ as the shared key with $U$.

In the second protocol instance, after receiving $m_{1}, S$ generates random number $t_{2}^{\prime} \in Z_{q}^{*}$, and sends $m_{2}^{\prime}=g^{t_{2}^{\prime}} \bmod p$ to $U$. $S$ also computes $z=g^{t_{2}^{t} t_{1}} \bmod p$ as the shared key material with $U$, and computes $K=h(z)$ as the shared key with $U$.

The attacker then intercepts and swaps these two messages, i.e., it impersonates $S$ to send $m_{2}^{\prime}$ and $m_{2}$ to $U$ in the first and second protocol instances respectively.

3. In the first protocol instance, after receiving $m_{2}^{\prime}, U$ computes $z=g^{t_{2}^{\prime} t_{1}}$ mod $p$ as the shared key material with $S$, and computes $K=h(z)$ as the shared key with $S$. Then $U$ constructs and sends the confirmation message $C_{1}=h(h(h(z)))$ to $S$.

In the second protocol instance, after receiving $m_{2}, U$ computes $z^{\prime}=$ $g^{t_{2} t_{1}^{\prime}} \bmod p$ as the shared key material with $S$, and computes $K^{\prime}=$ $h\left(z^{\prime}\right)$ as the shared key with $S$. Then $U$ constructs and sends the confirmation message $C_{1}^{\prime}=h\left(h\left(h\left(z^{\prime}\right)\right)\right)$ to $S$.

The attacker then intercepts and swaps these two messages, i.e., it impersonates $U$ to send $C_{1}$ and $C_{1}^{\prime}$ to $S$ in the second and first protocol instances respectively.

4. In the first protocol instance, after receiving $C_{1}^{\prime}, S$ checks that the received message equals $h\left(h\left(h\left(z^{\prime}\right)\right)\right)$. It is simple to verify that this check will succeed. $S$ computes and sends the confirmation message $C_{2}^{\prime}=h\left(h\left(z^{\prime}\right)\right)$ to $U$.

In the second protocol instance, after receiving $C_{1}, S$ checks that the received message equals $h(h(h(z)))$. It is simple to verify that this check will succeed. $S$ computes and sends the confirmation message $C_{2}=h(h(z))$ to $U$.

The attacker then intercepts and swaps these two messages, i.e., impersonates $S$ to send $C_{2}$ and $C_{2}^{\prime}$ to $U$ in the first second protocol instances respectively. 
5. In the first protocol instance, after receiving $C_{2}, U$ checks that it equals $h(h(z))$. $U$ has now confirmed that he shares the same key $K$ with $S$ in the first protocol instance, whereas $S$ computes the shared key as $K^{\prime}$ in the first protocol instance.

In the second protocol instance, after receiving $C_{2}^{\prime}, U$ checks that it equals $h\left(h\left(z^{\prime}\right)\right)$. $U$ has now confirmed that he shares the same key $K^{\prime}$ with $S$ in the second protocol instance, whereas $S$ computes the shared key as $K$ in the second protocol instance.

The above attack demonstrates that in any pair of concurrent protocol instances, beliefs of the participants regarding shared session keys can be falsified. In other words, in concurrent executions of the protocol an attacker can make the key confirmation progress give false results without it being detected by the participants.

The first password-based key agreement mechanism in [19] and the scheme BPKAS-SPEKE in [20] also suffer from this security vulnerability. Finally, note that this attack applies to many other two-party key agreement protocols, including those in $[19,20]$.

\section{Countermeasures}

The following methods can be used to prevent the two security vulnerabilities discussed above.

1. One possible method to prevent the first attack is to include the identities of the participants in the authentication messages $C_{1}$ and $C_{2}$. In the Jablon scheme, $C_{1}$ and $C_{2}$ would then be computed as follows:

$$
C_{1}=h\left(h\left(h\left(z|| I D_{U} \| I D_{S}\right)\right)\right), C_{2}=h\left(h\left(z|| I D_{S} \| I D_{U}\right)\right)
$$

Correspondingly, in the first password-based key agreement mechanism in [19], $C_{1}$ and $C_{2}$ would then be computed as follows:

$$
C_{1}=h\left(3\left\|m_{1}\right\| m_{2}\left\|g^{t_{1} t_{2}}\right\| g^{t_{1}}|| I D_{U} \| I D_{S}\right),
$$

and

$$
C_{2}=h\left(4|| m_{1}\left\|m_{2}\right\| g^{t_{1} t_{2}}\left\|g^{t_{1}}|| I D_{S}\right\| I D_{U}\right),
$$

2. One possible means preventing the second attack is including a unique session identifier in the computation of $g$ in every protocol instance. For example, in the two standardised mechanisms [19, 20] the session identifier could be included in str. 


\section{Conclusions}

In this paper we have shown that two potential security vulnerabilities exist in the strong password-only authenticated key exchange scheme due to Jablon [5]. The first password-based key agreement mechanism in [19], which is based on Jablon's scheme, suffers from both security vulnerabilities. Moreover, the other key agreement mechanisms in [19] also suffer from both security vulnerabilities. The scheme BPKAS-SPEKE in [20], which is also based on Jablon's scheme, suffers from the second security vulnerability. In fact, it might be possible to mount the second attack against many other two-party key agreement protocols.

\section{References}

[1] T. Lomas, L. Gong, J. Saltzer, and R. Needham. Reducing risks from poorly chosen keys. ACM SIGOPS Operating Systems Review, 23(5):14-18, 1989.

[2] S. M. Bellovin and M. Merritt. Encrypted key exchange: Passwordbased protocols secure against dictionary attacks. In SP '92: Proceedings of the 1992 IEEE Symposium on Security and Privacy, pages 72-84, Washington, DC, USA, 1992. IEEE Computer Society.

[3] S. M. Bellovin and M. Merritt. Augmented encrypted key exchange: A password-based protocol secure against dictionary attacks and password file compromise. In Proceedings of the First ACM Conference on Computer and Communications Security, pages 244-250, 1993.

[4] M. Steiner, G. Tsudik, and M. Waidner. Refinement and extension of encrypted key exchange. Operating Systems Review, 29(3):22-30, 1995.

[5] D. P. Jablon. Strong password-only authenticated key exchange. Computer Communication Review, 26(5):5-26, 1996.

[6] D. P. Jablon. Extended password key exchange protocols immune to dictionary attack. In Proceedings of the WETICE '97 Workshop on Enterprise Security, pages 248-255, Cambridge, MA, USA, 1997.

[7] S. Lucks. Open key exchange: How to defeat dictionary attacks without encrypting public keys. In B. Christianson, B. Crispo, T. Lomas, and M. Roe, editors, Proceedings of the 5th International Workshop on Security Protocols, pages 79-90. Springer-Verlag, Berlin, 1997.

[8] T. Wu. The secure remote password protocol. In Proceedings of the Network and Distributed System Security Symposium, NDSS 1998, pages 97-111, 1998. 
[9] O. Goldreich and Y. Lindell. Session-key generation using human passwords only. In J. Kilian, editor, Advances in Cryptology - CRYPTO '01, volume 2139 of Lecture Notes in Computer Science, pages 408-432. Springer-Verlag, 2001.

[10] M. Bellare, R. Canetti, and H. Krawczyk. A modular approach to the design and analysis of authentication and key exchange protocols (extended abstract). In STOC '98: Proceedings of the thirtieth annual ACM symposium on Theory of computing, pages 419-428, 1998.

[11] M. Bellare and P. Rogaway. Authenticated key exchange secure against dictionary attacks. Unpublished manuscript (service contribution) submitted to IEEE P1363, 2000.

[12] E. Bresson, O. Chevassut, and D. Pointcheval. New security results on encrypted key exchange. In B. Feng, R. Deng, and J. Zhou, editors, Proceedings of the 7th International Workshop on Theory and Practice in Public Key (PKC '04), volume 2947 of Lecture Notes in Computer Science, pages 145 -158. Springer-Verlag, 2004.

[13] T. Kwon. Practical authenticated key agreement using passwords. In K. Zhang and Y. Zheng, editors, Proceedings of the 7th Information Security Conference, volume 3225 of Lecture Notes in Computer Science, pages 1-12. Springer-Verlag, 2004.

[14] M. H. Nguyen and S. P. Vadhan. Simpler session-key generation from short random passwords. In M. Noar, editor, Proceedings of the First Theory of Cryptography Conference (TCC '04), volume 2951 of Lecture Notes in Computer Science, pages 428-445. Springer-Verlag, 2004.

[15] M. Abdalla, O. Chevassut, and D. Pointcheval. One-time verifier-based encrypted key exchange. In V. Serge, editor, Proceedings of the 8th International Workshop on Theory and Practice in Public Key (PKC '05), volume 3386 of Lecture Notes in Computer Science, pages 47-64. Springer-Verlag, 2005.

[16] M. Abdalla, P.-A. Fouque, and D. Pointcheval. Password-based authenticated key exchange in the three-party setting. In V. Serge, editor, Proceedings of the 8th International Workshop on Theory and Practice in Public Key (PKC '05), volume 3386 of Lecture Notes in Computer Science, pages 65-84. Springer-Verlag, 2005.

[17] M. Abdalla and D. Pointcheval. Simple password-based encrypted key exchange protocols. In A. Menezes, editor, Topics in Cryptology - CTRSA 2005, volume 3376 of Lecture Notes in Computer Science, pages 191-208. Springer-Verlag, 2005. 
[18] C. S. Laih, L. Ding, and Y. M. Huang. Password-only authenticated key establishment protocol without public key cryptography. Electronics Letters, 41(4):31-32, 2005.

[19] International Organization for Standardization. ISO/IEC FCD 117704, Information technology - Security techniques - Key management - Part 4: Mechanisms based on weak secrets, December 2004.

[20] Institute of Electrical and Electronics Engineers, Inc. IEEE P1363.2 draft D20, Standard Specifications for Password-Based Public-Key Cryptographic Techniques, March 2005. 